INFNCA-TH0204

MIT-CTP-3272

\title{
Two-dimensional dS/CFT correspondence
}

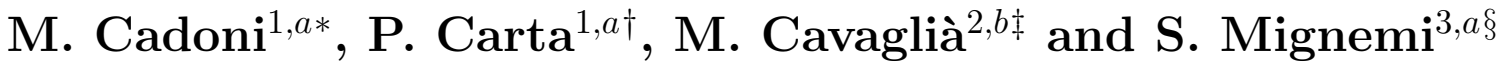 \\ ${ }^{1}$ Università di Cagliari, Dipartimento di Fisica, \\ Cittadella Universitaria, 09042 Monserrato, Italy \\ ${ }^{2}$ Center for Theoretical Physics, Massachusetts Institute of Technology, 77 Massachusetts \\ Avenue, Cambridge MA 02139-4307, USA \\ 3 Università di Cagliari, Dipartimento di Matematica, \\ Viale Merello 92, 09123 Cagliari, Italy \\ ${ }^{a}$ INFN, Sezione di Cagliari \\ ${ }^{b}$ INFN, Sede di Presidenza, Roma
}

\begin{abstract}
We investigate de Sitter/conformal field theory (dS/CFT) correspondence in two dimensions. We define the conserved mass of de Sitter spacetime and formulate the correspondence along the lines of anti-de Sitter/conformal field theory duality. Asymptotic symmetry group, mass, and central charge of de Sitter spacetime are equal to those of anti-de Sitter spacetime. The entropy of two-dimensional de Sitter spacetime is evaluated by applying Cardy formula. We calculate the boundary correlators induced by the propagation of the dilaton in two-dimensional de Sitter space. Although the dilaton is a tachyonic perturbation in the bulk, boundary conformal correlators have positive dimension.
\end{abstract}

*email: mariano.cadoni@ca.infn.it

†email: paolo.carta@ca.infn.it

‡email: cavaglia@mitlns.mit.edu

§email: smignemi@unica.it 


\section{Introduction}

Recently, Strominger proposed a correspondence between gravity on $d$-dimensional de Sitter space and $(d-1)$-dimensional conformal field theory [1], 2]. Evidence of a positive cosmological constant $\lambda$ provided by astrophysical observations [3, [4] suggests that we live in a de Sitter spacetime. An important feature of de Sitter spacetime is the existence of a cosmological horizon endowed with entropy [5]. De Sitter/Conformal Field Theory (dS/CFT) correspondence may hold the key to its microscopical interpretation. Moreover, new investigations have revealed the existence of holographic cosmological bounds on entropy and a correspondence between cosmological Friedmann equations and Cardy formula of CFT [6, 7]. $\mathrm{dS} / \mathrm{CFT}$ duality could be crucial in the understanding of the holographic principle in cosmology.

Naively, we would expect dS/CFT correspondence to proceed along the lines of Anti-de Sitter/Conformal Field Theory (AdS/CFT) correspondence because de Sitter spacetime can be obtained from anti-de Sitter spacetime by analytically continuing the cosmological constant to imaginary values. However, local and global properties of de Sitter spacetime lead to unexpected obstructions. Unlike anti-de Sitter, the boundary of de Sitter spacetime is spacelike and its dual CFT is Euclidean. Moreover, de Sitter spacetime does not admit a global timelike Killing vector. The time dependence of the spacetime metric precludes a consistent definition of energy and the use of Cardy formula to compute de Sitter entropy. Finally, dS/CFT duality leads to boundary operators with complex conformal weights, i.e., to a non-unitary CFT. In spite of these difficulties, some progress towards a consistent definition of $\mathrm{dS} / \mathrm{CFT}$ correspondence has been achieved. A new procedure [8] for the computation of the boundary stress tensor allows the definition of a conserved mass and the calculation of the entropy of asymptotically de Sitter spacetimes [8, 9]. In the three-dimensional case, by far the best-known example of the $\mathrm{dS}_{d} / \mathrm{CFT}_{d-1}$ correspondence, the central charge of the dual CFT has been computed and used in the Cardy formula to evaluate the entropy [1, 10, 11, 12.

In this paper we investigate $\mathrm{dS}_{d} / \mathrm{CFT}_{d-1}$ correspondence in two-dimensions. Previous investigations of $\mathrm{dS}_{2} / \mathrm{CFT}_{1}$ duality have only considered the quantization of scalar fields in two-dimensional (2D) de Sitter spacetime [13]. Here, we analyze the $\mathrm{dS}_{2} / \mathrm{CFT}_{1}$ correspondence in a full dynamical context, i.e., with 2D de Sitter spacetime emerging as a solution of the field equations. The main obstruction to the implementation of $\mathrm{dS}_{2} / \mathrm{CFT}_{1}$ correspondence along the lines of $\mathrm{AdS}_{2} / \mathrm{CFT}_{1}$ correspondence is the definition of a conserved mass for de Sitter spacetime. We show that a procedure similar to that of Ref. [8] enables the formulation of $\mathrm{dS}_{2} / \mathrm{CFT}_{1}$ correspondence in analogy to the $\mathrm{AdS}_{2} / \mathrm{CFT}_{1}$ case [14]. The generators of the asymptotic symmetric group of $\mathrm{dS}_{2}$ satisfy a Virasoro algebra. We compute the central charge of the algebra by adapting to $\mathrm{dS}_{2} / \mathrm{CFT}_{1}$ the canonical formalism of $\mathrm{AdS}_{2} / \mathrm{CFT}_{1}$ correspondence [14] and its interpretation as Casimir energy [15, 16]. The entropy of 2D de Sitter spacetime is evaluated by applying Cardy formula. In the second part of the paper we calculate the correlators induced on the one-dimensional boundary of the spacetime by the propagation of the dilaton in the 2D bulk. Although the dilaton is a tachyonic perturbation in the $2 \mathrm{D}$ spacetime, the dual 
boundary operator has positive conformal dimension $h=2$. This somehow unexpected result seems to be a general feature of the dS/CFT correspondence.

\section{2D Cosmological solutions of de Sitter gravity}

Let us consider the 2D dilaton gravity model with action

$$
I=\frac{1}{2} \int \sqrt{-g} d^{2} x \Phi\left(R-2 \lambda^{2}\right)
$$

where $\Phi$ is the dilaton field, $\lambda$ is the cosmological constant, and $R$ is the 2D Ricci scalar. The general solution of the model (1) describes a 2D hyperbolic manifold with constant positive curvature $R=2 \lambda^{2}$ (de Sitter spacetime) endowed with a non-constant dilaton. Two-dimensional de Sitter space can be defined as the hyperboloid

$$
X^{2}+Y^{2}-Z^{2}=\frac{1}{\lambda^{2}}
$$

embedded in the three-dimensional spacetime with hyperbolic metric $d s^{2}=d X^{2}+d Y^{2}-$ $d Z^{2}$. De Sitter spacetime can be interpreted as the analytical continuation $\lambda \rightarrow i \lambda$ of antide Sitter spacetime. De Sitter spacetime is geodesically complete. However, the presence of the dilaton field leads to three globally nonequivalent solutions which are described by coordinate charts covering different regions of the de Sitter hyperboloid. In analogy to the AdS case [17], we call these solutions $\mathrm{dS}_{0}, \mathrm{dS} \mathrm{S}_{-}, \mathrm{d \textrm {S } _ { + }}$ :

$\mathbf{d S}_{\mathbf{0}}$. In conformal coordinates the general solution of the gravity model (11) is

$$
d s^{2}=\frac{1}{\lambda^{2} \tau^{2}}\left(-d \tau^{2}+d x^{2}\right), \quad \Phi=\frac{\alpha\left(x^{2}-\tau^{2}\right)+\beta x+\gamma}{\tau},
$$

where $\alpha, \beta$, and $\gamma$ are integration constants. The spatial sections at $\tau=$ constant are either the line $(-\infty<x<\infty)$ or the one-dimensional sphere $S^{1}(-\pi<x<\pi)$. The solution (3) is singular at $\tau=0$. Therefore, $\mathrm{dS}_{0}$ covers half of the de Sitter hyperboloid (2). Setting $\lambda \tau=e^{\lambda T}$ the metric in Eq. (3) reads

$$
d s^{2}=-d T^{2}+e^{-2 \lambda T} d x^{2}
$$

where $-\infty<T<\infty$. Equation (44) is the $d=2$ case of the $\mathrm{dS}_{d}$ solution in planar coordinates [1, 2]. An interesting feature of the $2 \mathrm{D}$ solution is that the sections at $T=$ constant may have either the topology of the line or of the circle. In higher dimensions only planar topologies are allowed. The $(T, x)$ coordinate system covers half of the de Sitter hyperboloid. The spatial section at $T=-\infty(\tau=0)$ and $T=\infty(\tau=\infty)$ are the spacelike boundary $\mathcal{I}^{-}$of the spacetime and the cosmological future horizon, respectively. Alternatively, we can cover the other half of the de Sitter hyperboloid by setting $\lambda \tau=$ $-e^{-\lambda T}$. In this case $T=-\infty(\tau=-\infty)$ and $T=\infty(\tau=0)$ are the cosmological past 
horizon and the spacelike boundary $\mathcal{I}^{+}$of the spacetime. Setting $\lambda t=1 /(\lambda \tau)$ the line element in Eq. (3) becomes

$$
d s^{2}=-\frac{1}{\lambda^{2} t^{2}} d t^{2}+\lambda^{2} t^{2} d x^{2}
$$

The $\mathrm{dS}_{0}$ solution is the analytic continuation $\lambda \rightarrow i \lambda$ of the $2 \mathrm{D}$ anti-de Sitter $\mathrm{AdS}_{0}$ solution [17.

$\mathbf{d S}_{-}$. Setting

$$
\tau=\frac{1}{a \lambda} e^{a \lambda \sigma} \sinh (a \lambda \hat{\tau}), \quad x=\frac{1}{a \lambda} e^{a \lambda \sigma} \cosh (a \lambda \hat{\tau}),
$$

the metric in Eq. (3) is cast in the $\mathrm{dS}_{-}$form

$$
d s^{2}=\frac{a^{2}}{\sinh ^{2}(a \lambda \hat{\tau})}\left(-d \hat{\tau}^{2}+d \sigma^{2}\right) .
$$

The $(\hat{\tau}, \sigma)$ coordinates cover the region $x^{2} \geq \tau^{2}$ of $\mathrm{dS}_{0}$. The coordinate transformation (6) is analogous to the coordinate transformation which relates Minkowski and Rindler spacetimes. Curves of constant $\sigma$ are hyperbola in the $(\tau, x)$ coordinate frame and represent world lines of accelerated observers. Analogously to the AdS case [17], dS _ spacetime can be interpreted as the thermalization of $\mathrm{dS}_{0}$ spacetime at temperature $T_{H}=a \lambda / 2 \pi$. Defining

$$
\cosh \lambda T=\operatorname{cotanh}(a \lambda \hat{\tau})
$$

Eq. (7) reads:

$$
d s^{2}=-d T^{2}+a^{2} \sinh ^{2} \lambda T d \sigma^{2},
$$

where the cosmological time $T$ is defined in the interval $-\infty<T<0$. Equation (9) corresponds to the spherical slicing of higher dimensional de Sitter spacetime [1, 2]. Similarly to the planar slicing (4), the coordinate $\sigma$ can parametrize either a line or a circle. Setting $\lambda t=a \cosh \lambda T$, Eq. (9) is cast in the form

$$
d s^{2}=-\frac{1}{\lambda^{2} t^{2}-a^{2}} d t^{2}+\left(\lambda^{2} t^{2}-a^{2}\right) d \sigma^{2} .
$$

$\mathrm{dS}_{-}$can be interpreted as the analytic continuation, $\lambda \rightarrow i \lambda$, of the $2 \mathrm{D} \mathrm{AdS}_{+}$solution [17.

$\mathbf{d S}_{+}$. In conformal coordinates the $\mathrm{dS}_{+}$spacetime is described by the line element

$$
d s^{2}=\frac{a^{2}}{\cos ^{2}(a \lambda \tau)}\left(-d \tau^{2}+d \rho^{2}\right),
$$

where $-\pi / 2 a \leq \tau \leq \pi / 2 a$. Equation (11) describes the whole de Sitter hyperboloid (2). The spacelike coordinate $\rho$ can be either periodic or defined on the real line. Setting

$$
\sinh \lambda T=\tan (a \lambda \tau)
$$


Eq. (12) is cast in the form

$$
d s^{2}=-d T^{2}+a^{2} \cosh ^{2} \lambda T d \rho^{2},
$$

where $-\infty<T<\infty$. The spatial sections at $T= \pm \infty(\tau= \pm \pi / 2)$ are $\mathcal{I}^{+}$and $\mathcal{I}^{-}$, respectively. Finally, setting $\lambda t=a \sinh \lambda T$ the $\mathrm{dS}_{+}$line element becomes

$$
d s^{2}=-\frac{1}{a^{2}+\lambda^{2} t^{2}} d t^{2}+\left(a^{2}+\lambda^{2} t^{2}\right) d \rho^{2} .
$$

The $\mathrm{dS}_{+}$solution is the analytic continuation, $\lambda \rightarrow i \lambda$, of the $2 \mathrm{D}$ AdS - solution 17

\section{Isometries of $2 \mathrm{D}$ de Sitter spacetime and conserved mass}

The isometry group of 2D de Sitter spacetime is $S L(2, R)$. In the coordinate system of Eq. ([3) the isometry group of $\mathrm{dS}_{0}$ is described by the Killing vectors

$$
\xi_{0}=(\tau, x), \quad \xi_{1}=(0,2), \quad \xi_{-1}=\left(\tau x, \frac{1}{2}\left(\tau^{2}+x^{2}\right)\right) .
$$

The $S L(2, R)$ algebra is generated by the operators

$$
L_{0}=\tau \partial_{\tau}+x \partial_{x}, \quad L_{1}=2 \partial_{x}, \quad L_{-1}=\tau x \partial_{\tau}+\frac{1}{2}\left(\tau^{2}+x^{2}\right) \partial_{x} .
$$

$L_{0}, L_{1}$ and $L_{-1}$ generate dilatations, translations in $x$ and special conformal transformations, respectively. Any independent Killing vector defines an independent conserved charge. A crucial point is to identify the Killing vector that defines the energy of the solutions. Since we are dealing with time-dependent cosmological solutions, we do not expect any conserved charge associated with a globally timelike Killing vector. The Killing vectors $\xi_{1}$ and $\xi_{-1}$ are spacelike on the whole de Sitter hyperboloid. The Killing vector $\xi_{0}$ is timelike (spacelike) for $\tau^{2}>x^{2}\left(\tau^{2}<x^{2}\right)$. In particular, $\xi_{0}$ is spacelike on the boundaries $\mathcal{I}^{ \pm}$. As was pointed out in Ref. [1] for de Sitter spacetime in $d>2$ dimensions, the absence of a timelike conserved charge on the spacetime boundaries $\mathcal{I}^{ \pm}$represents

a serious obstruction to the implementation of the dS/CFT correspondence. For $d>2$ a solution to this problem has been proposed in Ref. [8], where the conserved mass of $\mathrm{dS}_{d}$ space is defined as an integral on the surface $\mathcal{S}$ orthogonal to a Killing vector $\tilde{\xi}$ of the boundary metric. This definition identifies the mass of the dS spacetime with the conserved charge of the theory living in its boundary. However, the procedure of Ref. [8] cannot be implemented in the $\mathrm{dS}_{2} / \mathrm{CFT}_{1}$ context because in this case the surface $\mathcal{S}$ is a point.

Using the results of Ref. [18], it is straightforward to prove that no timelike Killing vector exists on the spacetime boundary of $\mathrm{dS}_{2}$. Moreover, any Killing vector $\xi^{\nu}$ of the 
metric must also be a Killing vector of the dilaton field, i.e., $\xi^{\nu}$ must be a solution of the scalar Killing equation

$$
\xi^{\nu} \partial_{\nu} \Phi=0
$$

Given $\xi^{\nu}$, the quantity

$$
\mathcal{T}_{\mu}=T_{\mu \nu} \xi^{\nu}, \quad \nabla^{\mu} \mathcal{T}_{\mu}=0
$$

defines the conserved charge $Q$ through the equation $\mathcal{T}_{\mu}=\varepsilon_{\mu}^{\nu} \nabla_{\nu} Q$. In general, the dilaton gravity model (1) admits a Killing vector of the form

$$
\hat{\xi}^{\nu}=F_{0} \epsilon^{\nu \mu} \partial_{\mu} \Phi
$$

where $F_{0}$ is an arbitrary constant. The conserved charge is

$$
Q=\frac{F_{0}}{2}\left(-\lambda^{2} \Phi^{2}-(\nabla \Phi)^{2}\right)
$$

Equation (20) is a local and covariant definition of the conserved charge. Substituting the $\mathrm{dS}_{0}$ solution (3) in Eqs. (19) and (20) the Killing vector and charge read

$$
\begin{aligned}
\hat{\xi} & =F_{0}\left(2 \alpha x \tau+\beta \tau, \alpha\left(\tau^{2}+x^{2}\right)+\beta x+\gamma\right), \\
Q & =\frac{F_{0}}{2} \lambda^{2}\left(4 \alpha \gamma-\beta^{2}\right),
\end{aligned}
$$

respectively. As expected, $\hat{\xi}$ is a linear combination of the three Killing vectors of the metric (15). On the boundaries $\mathcal{I}^{ \pm}$the norm of $\hat{\xi}$ satisfies $\tau^{2}|\hat{\xi}|^{2}=(A+2 \gamma)^{2}$, where $A=x(\alpha x+\beta)$. Therefore, the Killing vector $\hat{\xi}^{\nu}$ is spacelike on $\mathcal{I}^{ \pm}$for any point of the moduli space. Moreover, there is no value of the parameters $\alpha, \beta$, and $\gamma$ such that $\hat{\xi}$ is everywhere timelike.

Although our model does not admit any global timelike Killing vector, Eqs. (21) and (22) define a one-to-one map between moduli space of the dilaton and symmetries and conserved charges. We can single out solutions with a given conserved charge by choosing the subgroup of $S L(2, R)$ that leaves the dilaton invariant. Solutions invariant under dilatations are obtained by choosing $\alpha=\gamma=0$. In this case the dilaton is

$$
\Phi=\beta \frac{x}{\tau},
$$

and the conserved charge under dilatations is $Q_{D}=-\frac{1}{2} F_{0}(\lambda \beta)^{2}$. We may also require that the dilaton depends only on time by setting $\alpha=\beta=0$. This singles out the $x$-translation generator from the $S L(2, R)$ isometry group of $\mathrm{dS}_{0}$.

Summarizing, we fix the charge $Q$ (up to a multiplicative constant) by choosing a point in the dilaton moduli space and identify the mass $M$ of the cosmological solution with $Q$ itself. This procedure is the 2D analogue of that of Ref. [8]: In two dimensions the Killing vector of the boundary metric is $\tilde{\xi} \propto \partial_{x}$ and the surface $\mathcal{S}$ which is orthogonal to $\tilde{\xi}$ is a point. The above procedure enables us to calculate the mass of $\mathrm{dS}_{0}, \mathrm{~d} \mathrm{~S}_{-}$and $\mathrm{d} \mathrm{S}_{+}$solutions. If we impose that the dilaton depends only on time, and use $t$ as timelike 
coordinate, $\Phi=\lambda^{2} \gamma t=\Phi_{0} \lambda t$ for the three different parametrizations (5), (10) and (14) of $\mathrm{dS}_{2}$. Using this equation in Eq. (20), we find $M=0$ and

$$
M= \pm F_{0} \frac{\lambda^{2}}{2} \Phi_{0}^{2} a^{2}
$$

for $\mathrm{dS}_{0}$ and $\mathrm{dS}_{ \pm}$, respectively. The mass (24) is defined up to the overall arbitrary constant $F_{0}$. The sign of $F_{0}$ can be fixed by requiring $M$ to be positive for the $\mathrm{dS}_{-}$solution ("stability" condition). The absolute value of $F_{0}$ is determined by requiring that $M$ coincides with the mass defined as a boundary integral (see Sect. 5). Together, these two conditions fix $F_{0}=-1 /\left(\lambda \Phi_{0}\right)$. With this choice the energy is positive, zero, and negative for $\mathrm{dS}_{-}, \mathrm{dS}_{0}$, and $\mathrm{dS}_{+}$, respectively. The Killing vector $\hat{\xi}$ is

$$
\hat{\xi}=(0,-1) \text {. }
$$

Translations in $x$ have opposite direction with respect to usual definition. With the above normalization the charge $Q$ of $\mathrm{dS}_{-}$is positive. The spacelike component of $\hat{\xi}$ and the stress energy-tensor are negative. The stability condition could also be enforced by keeping the usual definition of the Killing vector, $\hat{\xi}=(0,1)$, and reversing the sign of the action (and then of the stress-energy tensor $T_{\mu \nu}$ ). This arbitrariness indicates that $Q$ cannot be identified with the physical energy of the gravity theory in the $2 \mathrm{D}$ bulk.

\section{Asymptotic symmetries of 2D de Sitter spacetime}

Let us consider the $2 \mathrm{D}$ de Sitter solutions $\mathrm{dS}_{0}, \mathrm{dS} \mathrm{S}_{-}$and $\mathrm{dS}_{+}$. In the coordinate chart $(t, r)$, where $r=x, \sigma, \rho$ respectively for $\mathrm{dS}_{0}, \mathrm{dS}_{-}$and $\mathrm{dS}_{+}$, the Killing vectors generating the asymptotic symmetry group of the metric are

$$
\begin{aligned}
\xi^{t} & =-\epsilon^{\prime}(r) t+\frac{\alpha^{t}(r)}{t}+O\left(t^{-2}\right), \\
\xi^{r} & =\epsilon(r)+\frac{1}{2} \frac{\epsilon^{\prime \prime}(r)}{\lambda^{4} t^{2}}+\frac{\alpha^{r}(r)}{t^{4}}+O\left(t^{-5}\right) .
\end{aligned}
$$

The asymptotic form of the line element and of the dilaton which are invariant under the asymptotic symmetry group are 1

$$
\begin{aligned}
g_{t t} & =-\frac{1}{\lambda^{2} t^{2}}+\gamma_{t t}(r) \frac{1}{\lambda^{4} t^{4}}+O\left(t^{-5}\right), \\
g_{r r} & =\lambda^{2} t^{2}+\gamma_{r r}(r)+O\left(t^{-1}\right), \\
g_{t r} & =\frac{\gamma_{t r}(r)}{\lambda^{3} t^{3}}+O\left(t^{-4}\right), \\
\Phi & =\Phi_{0}\left[\lambda t+\rho(r) \lambda t+\gamma_{\phi \phi}(r) \frac{1}{\lambda t}+O\left(t^{-2}\right)\right] .
\end{aligned}
$$

\footnotetext{
${ }^{1}$ Analogously to the AdS case, the asymptotic form of the dilaton field is not invariant under the transformations generated by Eqs. (26) but changes with a term of the same order of the field itself.
} 
The asymptotic deformations of the fields transform as

$$
\begin{aligned}
\delta \rho & =\rho^{\prime} \epsilon-(1+\rho) \epsilon^{\prime}, \\
\delta \gamma_{\phi \phi} & =\gamma_{\phi \phi}^{\prime} \epsilon+\gamma_{\phi \phi} \epsilon^{\prime}+\frac{\rho^{\prime}}{2 \lambda^{2}} \epsilon^{\prime \prime}+\lambda^{2}(1+\rho) \alpha^{t}, \\
\delta \gamma_{t t} & =\gamma_{t t}^{\prime} \epsilon+2 \gamma_{t t} \epsilon^{\prime}+4 \lambda^{2} \alpha^{t}, \\
\delta \gamma_{r r} & =\gamma_{r r}^{\prime} \epsilon+2 \gamma_{r r} \epsilon^{\prime}+\frac{\epsilon^{\prime \prime \prime}}{\lambda^{2}}+2 \lambda^{2} \alpha^{t}, \\
\delta \gamma_{t r} & =\gamma_{t r}^{\prime} \epsilon+3 \gamma_{t r} \epsilon^{\prime}-\left(\gamma_{t t}+\gamma_{r r}\right) \frac{\epsilon^{\prime \prime}}{\lambda}-\lambda \alpha^{t^{\prime}}-4 \lambda^{5} \alpha^{r} .
\end{aligned}
$$

In analogy with the AdS/CFT correspondence, we can compute the generators of the asymptotic symmetry group. We must distinguish two cases, depending on whether the spacelike sections at $t=$ constant are the one-dimensional sphere $S^{1}(0 \leq r \leq 2 \pi / \lambda)$ or the real line $R(-\infty<r<\infty)$ :

$\mathbf{0} \leq \mathrm{r} \leq \mathbf{2 \pi} / \lambda$. For compact spatial sections $\epsilon$ can be expanded in Fourier series in the interval $[0,2 \pi / \lambda]$,

$$
\epsilon(r)=\sum_{k=0}^{\infty}\left[a_{k} \cos (\lambda k r)+b_{k} \sin (\lambda k r)\right] .
$$

The generators of the group of asymptotic symmetries are defined by

$$
\xi=\sum_{k=0}^{\infty} \lambda\left[a_{k} A_{k}+b_{k} B_{k}\right],
$$

where

$$
\begin{aligned}
& A_{k}=\left[k t+O\left(t^{-1}\right)\right] \sin (\lambda k r) \partial_{t}+\frac{1}{\lambda}\left(1-\frac{k^{2}}{2 \lambda^{2} t^{2}}+O\left(t^{-4}\right)\right) \cos (\lambda k r) \partial_{r}, \\
& B_{k}=\left[-k t+O\left(t^{-1}\right)\right] \cos (\lambda k r) \partial_{t}+\frac{1}{\lambda}\left(1-\frac{k^{2}}{2 \lambda^{2} t^{2}}+O\left(t^{-4}\right)\right) \sin (\lambda k r) \partial_{r} .
\end{aligned}
$$

The algebra of the $A_{k}$ and $B_{k}$ is

$$
\begin{aligned}
& {\left[A_{k}, A_{l}\right]=\frac{1}{2}(k-l) B_{k+l}+\frac{1}{2}(k+l) B_{k-l},} \\
& {\left[B_{k}, B_{l}\right]=-\frac{1}{2}(k-l) B_{k+l}+\frac{1}{2}(k+l) B_{k-l},} \\
& {\left[A_{k}, B_{l}\right]=-\frac{1}{2}(k-l) A_{k+l}+\frac{1}{2}(k+l) A_{k-l},}
\end{aligned}
$$

Defining the new generators $L_{k}=i A_{k}-B_{k}$ Eqs. (33) assume the standard form of a Virasoro algebra

$$
\left[L_{k}, L_{l}\right]=(k-l) L_{k+l}+\frac{c}{12}\left(k^{3}-k\right) \delta_{k+l},
$$

where we have taken into account the possibility of a central extension $c$. 
$-\infty<\mathbf{r}<\infty$. In this case $\epsilon$ is expanded in Laurent series

$$
\epsilon(r)=\sum_{k=-\infty}^{+\infty} a_{k}(\lambda r)^{k}
$$

The generators of the algebra are defined by

$$
\xi=\sum_{k=-\infty}^{+\infty} \lambda a_{k} \hat{L}_{k}
$$

where

$$
\hat{L}_{k}=\left[-k(\lambda r)^{k-1} t+O\left(t^{-1}\right)\right] \partial_{t}+\frac{1}{\lambda}\left[(\lambda r)^{k}+\frac{k(k-1)}{2 \lambda^{2} t^{2}}(\lambda r)^{k-2}+O\left(t^{-4}\right)\right] \partial_{r} .
$$

The algebra is

$$
\left[\hat{L}_{k}, \hat{L}_{m}\right]=(m-k) \hat{L}_{k+m-1} .
$$

Defining the new generators $L_{k}=-\hat{L}_{k+1}$, Eq. (38) is cast in the standard form (34).

\section{Central charge}

To calculate the central charge we use a canonical realization of the asymptotic symmetries. Since the boundary is spacelike, we parametrize the metric as

$$
d s^{2}=N^{2} d r^{2}-\Sigma^{2}\left(d t+N^{t} d r\right)^{2} .
$$

The 2D space is foliated along the spacelike coordinate $r$. Therefore, the dynamical evolution is generated by the Killing vector $\xi^{r}$. Owing to the normalization of $\xi^{r}$, Eq. (25), the integration measure along $r$ acquires an overall minus sign. Up to boundary terms the action becomes

$$
I=-\int d r d t\left[\frac{1}{N}\left(\Sigma^{\prime}-\frac{\partial}{\partial t}\left(N^{t} \Sigma\right)\right)\left(\Phi^{\prime}-N^{t} \dot{\Phi}\right)+N\left(\Sigma^{-1} \ddot{\Phi}-\Sigma^{-2} \dot{\Sigma} \dot{\Phi}-\lambda^{2} \Sigma \Phi\right)\right],
$$

where prime and dot denote differentiation with respect to $r$ and $t$, respectively. Introducing the conjugated momenta

$$
\Pi_{\Phi}=\frac{\delta \mathcal{L}}{\delta \Phi^{\prime}}, \quad \Pi_{\Sigma}=\frac{\delta \mathcal{L}}{\delta \Sigma^{\prime}},
$$

the action reads

$$
I=\int d r d t\left[\Pi_{\Sigma} \Sigma^{\prime}+\Pi_{\Phi} \Phi^{\prime}-N^{t} H^{t}-N H^{r}\right]
$$

where

$$
\begin{aligned}
& H^{t}=\Pi_{\Phi} \dot{\Phi}-\dot{\Pi}_{\Sigma} \Sigma \\
& H^{r}=-\Pi_{\Phi} \Pi_{\Sigma}+\Sigma^{-1} \ddot{\Phi}-\Sigma^{-2} \dot{\Sigma} \dot{\Phi}-\lambda^{2} \Sigma \Phi
\end{aligned}
$$


In order to have well-defined functional derivatives the Hamiltonian must be supplemented by the surface term $J$ :

$$
\mathcal{H}=\int d t\left(N^{t} H^{t}+N H^{r}\right)+J
$$

where

$$
\delta J=\lim _{t \rightarrow \infty}\left[N\left(\Sigma^{-2} \dot{\Phi} \delta \Sigma-\Sigma^{-1} \delta \dot{\Phi}\right)+\dot{N}\left(\Sigma^{-1} \delta \Phi\right)-N^{t}\left(\Pi_{\Phi} \delta \Phi-\Sigma \delta \Pi_{\Sigma}\right)\right] .
$$

In Eq. (45) we have considered only the contribution of the $t \rightarrow \infty$ boundary. The contribution of the $t=0$ boundary gives a similar contribution. We will come back later to this point.

Let us calculate the conserved charge $Q$ which is associated with the Killing vector $\partial_{r}$. The charge $Q$ will be identified with the mass of the solution. For the dS - solution (10), we have $\delta J=\delta Q=-(\lambda / 2) \Phi_{0} \delta \Sigma^{-2}$. It follows

$$
Q=\frac{\lambda}{2} \Phi_{0} a^{2}
$$

in agreement with Eq. (24). The mass of the $\mathrm{dS}_{+}$solution (14) is $Q=-(\lambda / 2) \Phi_{0} a^{2}$.

Using Eq. (26) and Eq. (27) in Eq. (45), the variations of the charges $J(\varepsilon)$ corresponding to the symmetries generated by the Killing vectors (26) are

$$
\delta J(\epsilon)=-\Phi_{0}\left(\epsilon \lambda\left(\gamma_{r r} \delta \rho-2 \delta \gamma_{\phi \phi}+\frac{1+\rho}{2} \delta \gamma_{t t}\right)+\frac{1}{\lambda}\left(\epsilon^{\prime \prime} \delta \rho-\epsilon^{\prime} \delta \rho^{\prime}\right)\right) .
$$

The central charge $c(\epsilon, \omega)$ can be calculated from the deformation algebra

$$
\delta_{\omega} J(\epsilon)=\{J(\epsilon), J(\omega)\}_{D B}=J([\epsilon, \omega])+c(\epsilon, \omega) .
$$

Substituting Eq. (47) in Eq. (48), and evaluating the equation on the $\mathrm{dS}_{0}$ background solution $\left(\rho=\gamma_{r r}=\gamma_{t t}=\gamma_{\phi \phi}=0\right.$ identically), we find

$$
c(\epsilon, \omega)=\frac{\Phi_{0}}{\lambda}\left(\epsilon^{\prime \prime} \omega^{\prime}-\epsilon^{\prime} \omega^{\prime \prime}\right)
$$

where we have used Eq. (28). Analogously to the 2D AdS case, the orthogonality problem 19, 14] can be solved by introducing the integrated charges (in this section we consider only $r$ periodic)

$$
\hat{J}(\epsilon)=\frac{\lambda}{2 \pi} \int_{0}^{2 \pi / \lambda} d r J(\epsilon) .
$$

The algebra (33) has central extension

$$
c\left(A_{k}, A_{l}\right)=c\left(B_{k}, B_{l}\right)=0, \quad c\left(A_{k}, B_{l}\right)=\Phi_{0} k^{2} l \delta_{|k||l|} .
$$

The central charge of the Virasoro algebra (34) is found by shifting the $L_{0}$ operator by a constant. The result is

$$
c=24 \Phi_{0}
$$


The central charge of de Sitter is positive and equal to that of anti-de Sitter. Following Ref. [20], we can integrate locally the variation (47) near the $\mathrm{dS}_{0}$ background solution: $J(\epsilon)=-\Phi_{0}\left(\epsilon^{\prime \prime} \rho-\epsilon^{\prime} \rho^{\prime}\right) / \lambda$. Since $J$ is defined up to a total $r$-derivative, it follows

$$
J(\epsilon)=-\frac{2 \Phi_{0}}{\lambda} \epsilon \rho^{\prime \prime}=\epsilon \Theta_{r r},
$$

where $\Theta_{r r}$ can be identified as the stress energy tensor of the one-dimensional boundary CFT. Using the transformation law of the boundary field $\rho$ we verify that $\Theta_{r r}$ transforms as a stress-energy tensor with central charge (52).

Up to now we have considered only the contribution of the boundary at $t=\infty$. By taking into account the contribution of the boundary at $t=0$ (see, e.g., Ref. [21]) the total central charge is

$$
c=12 \Phi_{0} .
$$

The result above can also be obtained by interpreting the central charge as Casimir energy. (This method was first used in Ref. [16] for 2D AdS/CFT correspondence and subsequently in Ref. [10 to calculate the central charge of three-dimensional de Sitter spacetime.) The $\mathrm{dS}_{-}$line element (10) is related to the $\mathrm{dS}_{0}$ line element (4) by the coordinate transformation

$$
e^{\lambda T}=\frac{e^{a \lambda \sigma}}{\sqrt{\lambda^{2} t^{2}-a^{2}}}, \quad x=\frac{t e^{a \lambda \sigma}}{a \sqrt{\lambda^{2} t^{2}-a^{2}}} .
$$

On the $t \rightarrow \infty$ boundary the coordinate transformation $x \rightarrow \sigma$ is

$$
x=\frac{e^{a \lambda \sigma}}{a \lambda} .
$$

Equation (56) is the one-dimensional analogue of the plane-cylinder transformation of a $2 \mathrm{D}$ conformal field theory. The stress-energy tensor $\Theta_{x x}$ acquires a term which is proportional to the central charge of the CFT and can be interpreted as a Casimir energy:

$$
\Theta_{x x}=\left(\frac{d x}{d \sigma}\right)^{2} \Theta_{\sigma \sigma}-\frac{c}{12}\left(\frac{d x}{d \sigma}\right)^{2}\{\sigma, x\},
$$

where $\{\sigma, x\}$ is the Schwarzian derivative. Substituting Eq. (56) in Eq. (57), and recalling that $\Theta_{\sigma \sigma}=-\lambda M=0\left(\Theta_{x x}=-\lambda M=-\frac{1}{2} \Phi_{0} a^{2} \lambda^{2}\right)$ for $\mathrm{dS}_{0}\left(\mathrm{dS}_{-}\right)$, we obtain the result (54).

\section{Entropy of de Sitter spacetime}

The solution (10) can be continued across the horizon $t^{2}<a^{2} / \lambda^{2}$ :

$$
d s^{2}=-\left(a^{2}-\lambda^{2} t^{2}\right) d \sigma^{2}+\frac{d t^{2}}{a^{2}-\lambda^{2} t^{2}}, \quad \Phi=\Phi_{0} \lambda t .
$$


Inside the horizon $t(\sigma)$ is spacelike (timelike). The metric is regular and admits the timelike Killing vector $\xi_{0}=\frac{\partial}{\partial \sigma}$. The dilaton has a naked timelike singularity at $t=0$, which is the lower-dimensional analogue of the conical singularity of three-dimensional de Sitter spacetime.

The temperature $T_{H}$ is the inverse of the period $\beta$ that must be assigned to the radial coordinate to avoid the conical singularity in the Euclidean section, i.e.,

$$
T_{H}=\left.\frac{1}{2 \pi} \frac{d g_{\sigma \sigma}}{d t}\right|_{\text {hor }}=\frac{\lambda a}{2 \pi} .
$$

The entropy can be calculated by using the Lorentzian action (see Ref. [9]). The Euclidean formalism of Gibbons and Hawking [5] is not suitable because the Euclidean action vanishes identically. The bulk term is identically zero on the field equations, and the Euclidean $\mathrm{dS}_{2}$ (two-sphere) has no boundary contribution. The Lorentzian action $I$ is 22 .

$$
I=\frac{1}{2} \int_{M} \sqrt{-g} d^{2} x \Phi\left(R-2 \lambda^{2}\right)+\int_{\partial M} \sqrt{h} d \sigma \Phi\left(K-K_{0}\right),
$$

where $h$ is the metric at the boundary, $K=-\frac{\dot{g}_{\sigma \sigma}}{2 \sqrt{g_{\sigma \sigma}}}$ is the trace of the extrinsic curvature evaluated at the boundary $t \rightarrow \infty$, and $K_{0}$ is the trace of the extrinsic curvature relative to the background metric $\mathrm{dS}_{0}$. The unusual sign in front of the boundary integral is due to the choice of normalization (25). Computing Eq. (60) on the solution (10), the boundary term gives $I=-\left(\beta \Phi_{0} \lambda a^{2}\right) / 2=-\pi \Phi_{0} a$. By analytically continuing the Gibbs-Duhem relation, we find

$$
S=\beta M-I=2 \pi \Phi_{0} a=2 \pi \Phi_{h},
$$

where $\Phi_{h}$ is the value of the dilaton at the horizon. Equation (61) is consistent with the thermodynamical relation $T_{H}=\partial M / \partial S$. The entropy can also be computed by applying Cardy formula to the boundary conformal field theory with central charge (54):

$$
S=2 \pi \sqrt{\frac{c l_{0}}{6}}=2 \pi \Phi_{0} a .
$$

Equation (62) is in agreement with the semiclassical result (61).

\section{Boundary correlators}

In this section we discuss $\mathrm{dS}_{2} / \mathrm{CFT}_{1}$ correspondence by computing correlation functions on the spacetime bulk and on its boundary. In higher-dimensional de Sitter spacetimes this program is accomplished by studying correlation functions of dual boundary operators induced by an external field. The 2D model (11), thanks to the presence of a scalar degree of freedom (the dilaton field $\Phi$ ), enables to compute correlation functions which are induced on the boundary by the gravitational degrees of freedom of the bulk. Let us consider the field equations of the dilaton

$$
\nabla_{\mu} \nabla_{\nu} \Phi=-\lambda^{2} g_{\mu \nu} \Phi
$$


2D dilaton gravity has no propagating physical degrees of freedom: If we restrict ourselves to classical configurations, and fix the diffeomorphism invariance of the theory, the dilaton does not propagate. However, we allow dilaton deformations on the one-dimensional boundary of $\mathrm{dS}_{2}$ ( $\rho$ and $\gamma_{\phi \phi}$ fields in Eq. (27)). These deformations correspond to pure gauge and off-shell dilaton propagation on the spacetime boundary. Therefore, we require that the dilaton satisfies the trace equation

$$
\nabla^{2} \Phi=-2 \lambda^{2} \Phi
$$

instead of the full equations of motion (63). Equation (64) is the equation of motion of a scalar field with negative mass-squared $m^{2}=-2 \lambda^{2}$ and describes the propagation of a tachyonic scalar field in 2D de Sitter spacetime. Consider Eqs. (四), (9) and (13), where $r=x, \sigma$, and $\rho$, respectively. Owing to the presence of the cosmological horizon, no correlators between $r \in \mathcal{I}^{-}$and $r^{\prime} \in \mathcal{I}^{+}$exist for $\mathrm{dS}_{0}$ and $\mathrm{dS}_{-}$. On the contrary, $\mathrm{dS}_{+}$covers the whole de Sitter spacetime and non-trivial correlators between $r \in \mathcal{I}^{-}$and $r^{\prime} \in \mathcal{I}^{+}$exist. Let us deal with the three cases separately:

$\mathbf{d S}_{\mathbf{0}}$. In the background (4) Eq. (64) reads

$$
\left(-\partial_{T}^{2}+\lambda \partial_{T}+e^{2 \lambda T} \partial_{r}^{2}\right) \Phi=-2 \lambda^{2} \Phi
$$

When $T \rightarrow-\infty$, the third term on the left hand side of Eq. (65) is negligible. On the $\mathcal{I}^{-}$ boundary the dilaton is

$$
\Phi \sim \phi_{-1}(r) e^{-\lambda T}
$$

Subleading terms can be evaluated by expanding $\Phi$ in powers of $e^{\lambda T}$ :

$$
\Phi=\sum_{n=-1}^{\infty} \phi_{n}(r) e^{n \lambda T} .
$$

Substituting Eq. (67) in Eq. (65) we find

$$
\Phi=\phi_{-1}(r) e^{-\lambda T}+\phi_{1}(r) e^{\lambda T}+O\left(e^{2 \lambda T}\right)
$$

where the index denotes the conformal dimension of the fields $\phi . \mathrm{dS}_{0}$ has no boundary fields with conformal dimensions $h=0$. The conformal weights of subleading and leading terms in Eq. (68) are consistent with the conformal transformation laws of $\rho$ and $\gamma_{\phi \phi}$ with weights $h= \pm 1$, respectively. It is interesting to compare the boundary condition (68) with that of a generic scalar field of mass $m$ that propagates on $2 \mathrm{D}$ de Sitter spacetime [13]: $\Phi \sim e^{h_{ \pm} \lambda T}$, where $h_{ \pm}=\left(1 \pm \sqrt{1-4 m^{2} / \lambda^{2}}\right) / 2$. Setting $m^{2}=-2 \lambda^{2}$ we find $h_{+}=-1$ and $h_{-}=2$. However, the previous result is only valid for scalar fields with positive squared mass. For tachyonic fields we have $h_{+}>1$ and the term of weight $h_{+}$is subleading with respect to $\phi_{1}$.

Changing the sign in the exponents of Eqs. (4), (65) and (66) we obtain the behavior of the dilaton on the boundary $\mathcal{I}^{+}$:

$$
\Phi=\phi_{1}(r) e^{\lambda T}+\phi_{-1}(r) e^{-\lambda T}+O\left(e^{-2 \lambda T}\right)
$$


Leading and subleading terms of the dilaton on $\mathcal{I}^{+}$are interchanged with respect to $\mathcal{I}^{-}$. Generalizing to two-dimensions the dS/CFT proposal of Ref. [1], the two-point correlator of an operator $\mathcal{O}_{\phi}$ on $\mathcal{I}^{-}$is derived from the expression

$$
\mathcal{J}=\lim _{T \rightarrow-\infty} \int_{\mathcal{I}^{-}} d r d r^{\prime}\left[e^{-\lambda\left(T+T^{\prime}\right)} \Phi(T, r) \stackrel{\leftrightarrow}{\partial}_{T} G\left(T, r, T^{\prime}, r^{\prime}\right) \stackrel{\leftrightarrow}{\partial}_{T^{\prime}} \Phi\left(T^{\prime}, r^{\prime}\right)\right]_{T=T^{\prime}},
$$

where $G$ is the de Sitter invariant Green function. (See Appendix.) Using Eqs. (85), (90) and (68) in Eq. (70), we find

$$
\mathcal{J}=\kappa_{0} \int_{\mathcal{I}^{-}} d r d r^{\prime} \phi_{-1}(r) \phi_{-1}\left(r^{\prime}\right) \frac{1}{\left(r-r^{\prime}\right)^{4}},
$$

where $\kappa_{0}$ is a constant. The two-point correlator of an operator $\mathcal{O}_{\phi}$ dual to $\phi_{-1}$ is the coefficient of the quadratic term in Eq. (71):

$$
\left\langle\mathcal{O}_{\phi}(r) \mathcal{O}_{\phi}\left(r^{\prime}\right)\right\rangle=\frac{\kappa_{0}^{\prime}}{\left(r-r^{\prime}\right)^{4}} .
$$

Equation (72) is the two-point correlator of a conformal operator of dimension $h=2$. The two-point correlator on $\mathcal{I}^{+}$can be computed in a similar way. The relevant boundary field is $\phi_{1}(r)$ and the dual operator on $\mathcal{I}^{+}$satisfies Eq. (72). The previous results show that a tachyonic perturbation of the bulk corresponds to a boundary operator of positive conformal dimension. This feature is a consequence of the holographic correspondence between gravity on the 2D bulk and CFT on the boundary. Technically, the result follows from a general property of the integral in Eq. (70). The dual operator of a boundary field with conformal dimension $h_{-}$has dimension $h_{+}$. Therefore, the tachyonic perturbation $\left(h_{-}<0\right)$ is in correspondence with a boundary operator of positive conformal dimension $\left(h_{+}>0\right)$. This property seems to be a general feature of the integral (70) and we expect it to hold for dS/CFT duality in any dimension.

$\mathbf{d S}_{-}$. In this case de Sitter spacetime is described by the metric (9). Equation (64) is

$$
\left(-\partial_{T}^{2}-\lambda \operatorname{coth}(\lambda T) \partial_{T}+\frac{1}{a^{2} \sinh ^{2}(\lambda T)} \partial_{r}^{2}\right) \Phi=-2 \lambda^{2} \Phi .
$$

Setting $T \rightarrow \pm \infty$ in Eq. (73), we find that the asymptotic behavior of the dilaton is given by Eqs. (68) and (69) for $\mathcal{I}^{-}$and $\mathcal{I}^{+}$, respectively. The dS- boundary correlators are computed by substituting Eqs. (68) and (69) and the asymptotic expression of $G$ and $P$ given in the Appendix in the integral (70):

$$
\left\langle\mathcal{O}_{\phi}(r) \mathcal{O}_{\phi}\left(r^{\prime}\right)\right\rangle=\frac{\kappa_{-}}{\sinh ^{4} \frac{a \lambda}{2}\left(r-r^{\prime}\right)} .
$$

Since $\mathrm{dS}_{0}$ and $\mathrm{dS}_{-}$are locally identical, the correlators (72) and (74) have the same $\Delta r=r-r^{\prime} \rightarrow 0$, short-distance, behavior. The global features of the spacetime become 
manifest at large $\Delta r$. The sinh behavior in Eq. (74) describes a thermal CFT with temperature equal to the Hawking temperature of the cosmological horizon of dS_. This result can be explained in CFT as follows. Equation (56) maps the boundary of $\mathrm{dS}_{0}$ on the boundary of $\mathrm{dS}_{-}$. This transformation can be interpreted as the one-dimensional analogue of the plane-cylinder map $\lambda z=\exp (\lambda w)$ of a 2D CFT, where $w=-\bar{w}=-i r$. In complex coordinates Eq. (74) becomes

$$
\left\langle\mathcal{O}_{\phi}(r) \mathcal{O}_{\phi}\left(r^{\prime}\right)\right\rangle=\frac{\kappa_{-}^{\prime}}{\left[\sin \left(\pi T_{H} \Delta w\right) \sin \left(\pi T_{H} \Delta \bar{w}\right)\right]^{2}},
$$

where $\Delta w=w-w^{\prime}$ and $T_{H}$ is the Hawking temperature of the cosmological horizon (59). The appearance of thermal correlators can also be understood in terms of the $2 \mathrm{D}$ gravity theory: $\mathrm{dS}_{-}$can be considered as the thermalization of $\mathrm{dS}_{0}$ at temperature $T_{H}=\lambda a / 2 \pi$ (see Sect. 2).

$\mathbf{d S}_{+}$. In the background (13) the equation of motion of the dilaton is

$$
\left(-\partial_{T}^{2}-\lambda \tanh (\lambda T) \partial_{T}+\frac{1}{a^{2} \cosh ^{2}(\lambda T)} \partial_{r}^{2}\right) \Phi=-2 \lambda^{2} \Phi
$$

On the spacetime boundaries $\mathcal{I}^{-}$and $\mathcal{I}^{+}$we obtain again Eqs. (68) and (69), respectively. Using Eqs. (85) and (90) of the Appendix, the two-point boundary correlator is

$$
\left\langle\mathcal{O}_{\phi}(r) \mathcal{O}_{\phi}\left(r^{\prime}\right)\right\rangle=\frac{\kappa_{+}}{\sin ^{4} \frac{a \lambda}{2}\left(r-r^{\prime}\right)},
$$

where $r, r^{\prime} \in \mathcal{I}^{-}$or $r, r^{\prime} \in \mathcal{I}^{+}$. Equation (77) is the correlator for an operator of conformal dimension $h=2$. In the $\mathrm{dS}_{+}$case we must also consider correlators between points $r, r^{\prime}$, where $r \in \mathcal{I}^{-}$and $r^{\prime} \in \mathcal{I}^{+}$. This corresponds to let $T \rightarrow-\infty$ and $T^{\prime} \rightarrow \infty$ in Eq. (70). The function $P$ defined in Eq. (84) satisfies the equation

$$
P\left(T, r, T^{\prime}, r^{\prime}\right)=-P\left(T, r,-T^{\prime}, r^{\prime}+\pi\right) .
$$

Using Eq. (78) for $r \in \mathcal{I}^{-}$and $r^{\prime} \in \mathcal{I}^{+}, \mathcal{J}$ becomes

$$
\mathcal{J}=\kappa_{+}^{\prime} \int d r d r^{\prime} \phi_{-1}(r) \phi_{-1}\left(r^{\prime}+\pi\right) \frac{1}{\sin ^{4} \frac{a \lambda}{2}\left(r-r^{\prime}\right)} .
$$

Analogously to the three-dimensional case of Ref. [1], we can define the inverted boundary field $\tilde{\phi}_{-1}(r)=\phi_{-1}(r+\pi)$ and find the non-trivial correlations between points of the two different boundaries. The correlator of boundary operators dual to $\tilde{\phi}_{-1}\left(r^{\prime}\right)$ and $\phi_{-1}(r)$ coincide with the two-point correlator on a single boundary (77).

\section{Conclusions}

In this paper we have investigated the $2 \mathrm{D}$ dS/CFT correspondence. The de Sitter conserved mass has been defined by exploiting a peculiar feature of 2D dilaton gravity, namely 
the existence of a locally defined, general covariant conserved charge. The $\mathrm{dS}_{2} / \mathrm{CFT}_{1} \mathrm{du}-$ ality has been implemented in analogy with the $\mathrm{AdS}_{2} / \mathrm{CFT}_{1}$ correspondence. We have shown that the group of the asymptotic symmetries of $\mathrm{dS}_{2}$ is equal to that of $\mathrm{AdS}_{2}$ and is generated by the same Virasoro algebra. The statistical entropy of the de Sitter cosmological horizon coincides with the statistical entropy of the AdS black hole. These results follow from the interpretation of de Sitter spacetime as "Wick rotated" AdS spacetime. Similar conclusions have been obtained for higher-dimensional de Sitter spacetime in Refs. 11, 9, 12, 10]. A major difference between 2D de Sitter spacetime and the higherdimensional cases is the absence, in the former, of an upper bound for the entropy. The entropy (61) grows without limit with $a$. The origin of the difference can be understood by comparing de Sitter in two and three dimensions. The behavior of the entropy as a function of $a$ in $d=2$ and $d=3$ is identical. However, the presence of the conical singularity in $d=3$ provides the upper bound $a=1$. For $d=2$ the spacelike coordinate $r$ is not a radial coordinate. Therefore, no "natural" normalization can be imposed on it. In Ref. [7] it was argued that this feature follows from the symmetry under dilatations of the model and is related with the impossibility of establishing an area law in two dimensions.

In the second part of the paper we have computed the boundary correlators of the model. We have found that the dS/CFT correspondence leads to boundary operators of positive conformal dimension for 2D bulk tachyonic perturbations. Non-causal tachyonic propagation in the bulk are usually expected to lead to boundary operators with negative dimension, i.e., to correlators which usually describe a non-unitary CFT, such as $\left\langle\mathcal{O}(r) \mathcal{O}\left(r^{\prime}\right)\right\rangle \sim\left(r-r^{\prime}\right)^{l}$, where $l>0$. The positivity of the conformal dimension seems to be strongly related to the holographic nature of the $\mathrm{dS} / \mathrm{CFT}$ correspondence. It would be very interesting to understand whether this property is a peculiarity of the $2 \mathrm{D}$ case or a general feature of the dS/CFT duality.

\section{Appendix}

In this appendix we derive the 2D, de Sitter-invariant, Hadamard two-point function for the dilaton field

$$
G\left(X, X^{\prime}\right)=\left\langle 0\left|\left\{\Phi(X), \Phi\left(X^{\prime}\right)\right\}\right| 0\right\rangle .
$$

Equation (80) is solution of the equation

$$
\left(\nabla_{X}^{2}-m^{2}\right) G\left(X, X^{\prime}\right)=0
$$

where $m^{2}=-2 \lambda^{2}$. Analogously to higher-dimensional cases, the $S O(1,1)$ invariant Green function can only be a function of the geodesic distance $d\left(X, X^{\prime}\right)$, where $X, X^{\prime}$ are coordinates of the three-dimensional Minkowski embedding spacetime. It is convenient to introduce the quantity

$$
P\left(X, X^{\prime}\right)=\lambda^{2} X^{A} X^{\prime B} \eta_{A B},
$$

where $P=\cos \lambda d$ and $\eta_{A B}=(1,1,-1)$ is the metric of the three-dimensional Minkowski spacetime. Using Eq. (2) $P$ can be calculated for the three different parametrizations of 
$\mathrm{dS}_{2}$. We have

$$
\begin{aligned}
\mathrm{dS}_{0}: & \lambda X=e^{-\lambda T} \lambda r, \lambda Y=\cosh \lambda T-e^{\lambda T}(\lambda r)^{2} / 2, \lambda Z=-\sinh \lambda T+e^{-\lambda T}(\lambda r)^{2} / 2, \\
\mathrm{dS}_{-}: & \lambda X=\cosh \lambda T, \quad \lambda Y=\sinh \lambda T \sinh \lambda r, \quad \lambda Z=\sinh \lambda T \cosh \lambda r, \\
\mathrm{dS}_{+}: & \lambda X=\cosh \lambda T \sin \lambda r, \quad \lambda Y=\cosh \lambda T \cos \lambda r, \quad \lambda Z=\sinh \lambda T .
\end{aligned}
$$

Substituting Eqs. (83) in Eq. (82) we find

$$
\begin{array}{ll}
\mathrm{dS}_{0}: & P=\cosh \lambda\left(T-T^{\prime}\right)-\lambda^{2} e^{-\lambda\left(T+T^{\prime}\right)}\left(r-r^{\prime}\right)^{2} / 2, \\
\mathrm{dS}_{-}: & P=\cosh \lambda T \cosh \lambda T^{\prime}-\sinh \lambda(T) \sinh \lambda T^{\prime} \cosh \lambda\left(r-r^{\prime}\right), \\
\mathrm{dS}_{+}: & P=\cosh \lambda T \cosh \lambda T^{\prime} \cos \lambda\left(r-r^{\prime}\right)-\sinh \lambda T \sinh \lambda T^{\prime} .
\end{array}
$$

The asymptotic behavior of $P$ at $T \rightarrow-\infty$ is

$$
\begin{aligned}
\mathrm{dS}_{0}: & \lim _{T, T^{\prime} \rightarrow-\infty} P=-\frac{\lambda^{2}}{2} e^{-\lambda\left(T+T^{\prime}\right)}\left(r-r^{\prime}\right)^{2}, \\
\mathrm{dS}_{-}: & \lim _{T, T^{\prime} \rightarrow-\infty} P=-\frac{1}{2} e^{-\lambda\left(T+T^{\prime}\right)} \sinh ^{2} \lambda \frac{\left(r-r^{\prime}\right)}{2}, \\
\mathrm{dS}_{+}: & \lim _{T, T^{\prime} \rightarrow-\infty} P=-\frac{1}{2} e^{-\lambda\left(T+T^{\prime}\right)} \sin ^{2} \lambda \frac{\left(r-r^{\prime}\right)}{2} .
\end{aligned}
$$

The behavior of $P$ on $\mathcal{I}^{+}$can be obtained by changing the signs of $T$ and $T^{\prime}$ in Eqs. (85). For a generic scalar field $\phi$ of mass $m$ propagating in 2D de Sitter spacetime, Eq. (81) is [2, 23]

$$
\left[\left(1-P^{2}\right) \frac{d^{2}}{d P^{2}}-2 P \frac{d}{d P}-\frac{m^{2}}{\lambda^{2}}\right] G(P)=0 .
$$

The solution of Eq. (86) is

$$
G=\operatorname{ReF}\left(h_{+}, h_{-}, 1, z\right),
$$

where $\mathrm{F}$ is the hypergeometric function, $z=(1+P) / 2$, and $h_{ \pm}=\left(1 \pm \sqrt{1-4 m^{2} / \lambda^{2}}\right) / 2$. For the dilaton field $\left(m^{2}=-2 \lambda^{2}\right)$ the general solution of Eq. (86) can be expressed in terms of elementary functions:

$$
G(P)=c_{1}\left(2-P \ln \left|\frac{P+1}{P-1}\right|\right)+c_{2} P,
$$

where $c_{1}, c_{2}$ are integration constants. The Green function $G$ is the sum of two independent terms. The second term grows linearly with $P$ and is singular in the $P \rightarrow \infty$ limit. This leads to divergences in Eq. (70). The singularity can be removed by imposing the boundary condition $c_{2}=0$. (It is not clear whether other physically acceptable boundary conditions exist). The first term in Eq. (88) describes a Green function with two singularities at $P= \pm 1$. The behavior at short distances is that of a scalar field in a $2 \mathrm{D}$ spacetime. Near $P=1$, i.e., at a geodesic distance $d=0, G$ is

$$
\lim _{P \rightarrow 1} G=2 c_{1} \ln (\lambda d) .
$$


The integration constant $c_{1}$ can be determined by comparing Eq. (89) to the usual shortdistance behavior in two-dimensions $G=-1 / 2 \pi \ln (\lambda d)$. The behavior at $P \rightarrow \infty$ is

$$
G(P)=-\frac{2}{3} \frac{c_{1}}{P^{2}}+O\left(P^{-4}\right) .
$$

Finally, the $\left(c_{2}=0\right)$ Green function is an even function of $P$.

\section{References}

[1] A. Strominger, JHEP 0110, 034 (2001) arXiv:hep-th/0106113.

[2] M. Spradlin, A. Strominger and A. Volovich, arXiv:hep-th/0110007.

[3] S. Church, A. Jaffe and L. Knox, arXiv:astro-ph/0111203.

[4] A. Albrecht, J. A. Frieman and M. Trodden, in Proc. of the APS/DPF/DPB Summer Study on the Future of Particle Physics (Snowmass 2001) ed. R. Davidson and C. Quigg, arXiv:hep-ph/0111080.

[5] G. W. Gibbons and S. W. Hawking, Phys. Rev. D 15, 2738 (1977).

[6] E. Verlinde, arXiv:hep-th/0008140.

[7] M. Cadoni, P. Carta and S. Mignemi, arXiv:hep-th/0202180.

[8] V. Balasubramanian, J. de Boer and D. Minic, arXiv:hep-th/0110108.

[9] A. M. Ghezelbash and R. B. Mann, JHEP 0201, 005 (2002) arXiv:hep-th/0111217.

[10] D. Klemm, Nucl. Phys. B 625, 295 (2002) arXiv:hep-th/0106247.

[11] J. M. Maldacena and A. Strominger, JHEP 9802, 014 (1998) arXiv:gr-qc/9801096.

[12] Y. S. Myung, Mod. Phys. Lett. A 16, 2353 (2001) arXiv:hep-th/0110123.

[13] S. Ness and G. Siopsis, arXiv:hep-th/0202096.

[14] M. Cadoni and S. Mignemi, Nucl. Phys. B 557, 165 (1999) arXiv:hep-th/9902040.

[15] M. Cadoni and M. Cavaglià, Phys. Rev. D 63, 084024 (2001) arXiv:hep-th/0008084.

[16] M. Cadoni and M. Cavaglià, Phys. Lett. B 499, 315 (2001) arXiv:hep-th/0005179.

[17] M. Cadoni and S. Mignemi, Phys. Rev. D 51, 4319 (1995) arXiv:hep-th/9410041.

[18] R. B. Mann, Phys. Rev. D 47, 4438 (1993) arXiv:hep-th/9206044].

[19] S. Carlip, Class. Quant. Grav. 16, 3327 (1999) arXiv:gr-qc/9906126]. 
[20] M. Cadoni and S. Mignemi, Phys. Lett. B 490, 131 (2000) [arXiv:hep-th/0002256.

[21] M. Cadoni, P. Carta, D. Klemm and S. Mignemi, Phys. Rev. D 63, 125021 (2001) arXiv:hep-th/0009185.

[22] J. W. York, Found. Phys. 16, 249 (1986).

[23] P. Candelas and D. J. Raine, Phys. Rev. D 12, 965 (1975). 